\title{
A novel AGC simulation scheme based on reduced order prime mover models
}

\author{
K. C. Divya and P. S. Nagendra Rao \\ Department of Electrical Engineering, \\ Indian Institute of Science, \\ Bangalore 560012, India. \\ divya,nagendra@ee.iisc.ernet.in
}

\begin{abstract}
,Abstract-This paper presents a new simulation scheme for AGC studies of power system. The proposed scheme uses a different set of assumptions from that conventionally used. In this scheme, all the areas have been considered to he operating at the same frequency. Further, the proposed approach can preserve the identity of each generating unit. Additionally, the computational complexity has been reduced by resorting to lower order generating unit models. In this context, model order reduction techniques have been used to obtain lower order models for AGC studies. The effectiveness of the new simulation approach is demonstrated by considering the IEEE 30 bus test system as a $\mathbf{3}$ area system.
\end{abstract}

\section{INTRODUCTION}

AGC is an important control problem encountered in interconnected power systems. Most of the AGC study is concerned with the design of different types of controllers and validating the design using the power system models commonly available in the literature.'

The power system models that are extensively used in the AGC literature require : i) identitication of control areas. ii) aggregate model to represent the area generator unit dynamics. iii) computation of the tie line constant, in order to compute the tie line flows. The above three modelling steps can be adopted where in the control area consists of a group of coherent generators. This situation is however not commonly encountered in practice. The control area boundaries get detined from the commercial boundaries. If the units within the area are not coherent then it is difficult to identify each control area with a set of coherent generators. Hence, obtaining an aggregate model to represent the area dynamics will also be difficult. Additionally, the use of aggregate model causes the identity of each generator to be lost, which would make it difficult to take into account the non-linearities (GRC and dead band) of each unit. Apart from this, there are no well defined methods available, at present, to compute the tie line constant for an area with non-coherent generators and multiple tie lines.

The aim of this paper is to evolve an AGC simulation scheme that overcomes the above mentioned limitations. In the proposed scheme, it has been assumed that all the areas of the

\footnotetext{
0-7803-7651-X/03/\$17.00 @2013 EFEE
}

system operate at the same frequency. Though this assumption has not been made in earlier AGC studies, it has been recommended in [1] and also by the IEEE committee [2]. This assumption eliminates the need to identify coherent set of generators in order to identify the control areas. Hence. in this scheme of simulation the control areas are identitied purely by the commercial boundaries.

The other feature of this assumption is that it eliminates the need to compute the tie line constant. This follows from the fact that all the areas are operating at the same frequency and the tie line flows can no longer,be computed in the conventional method (i.e. based on the difference in area angles and the tie line constant). However, as the tie line flows are needed to compute the ACE, the use of an alternative approach, viz. energy balance equation, has been proposed here.

Further, in the proposed method the identity of each generating unit can be preserved unlike in the available methods which. use an aggregate area model. Although this feature is desirable, it results in a substantial increase in the computational effort, especially for a practical system. In order to over come this difficulty, the use of reduced order models for the generating units (prime mover) has been proposed here. The reduced order models for these prime movers have been obtained by using some of the well known model order reduction techniques. These reduced order models have been validated with that of the full order models, by comparing their response. Further, the utility of these lower order prime mover models for AGC studies has been investigated. This has been done by first comparing the primary responses of the full and reduced order models. Also the amenability of the reduced order models for AGC design has been demonstrated by designing an integral controller.

\section{PROPOSED AGC SIMULATION SCHEME}

The general structúre of the simulation model proposed for AGC studies has been shown in Fig 1. The use of this type of simulation model for AGC studies has been justified in [1] and [2], where in it has been stated that AGC is, a slow process and any AGC simulation model must capture only the slow dynamics of the system.

In [1] and [2] it has also been suggested that the intermachine 


\section{TENCON $2003 / 1100$}

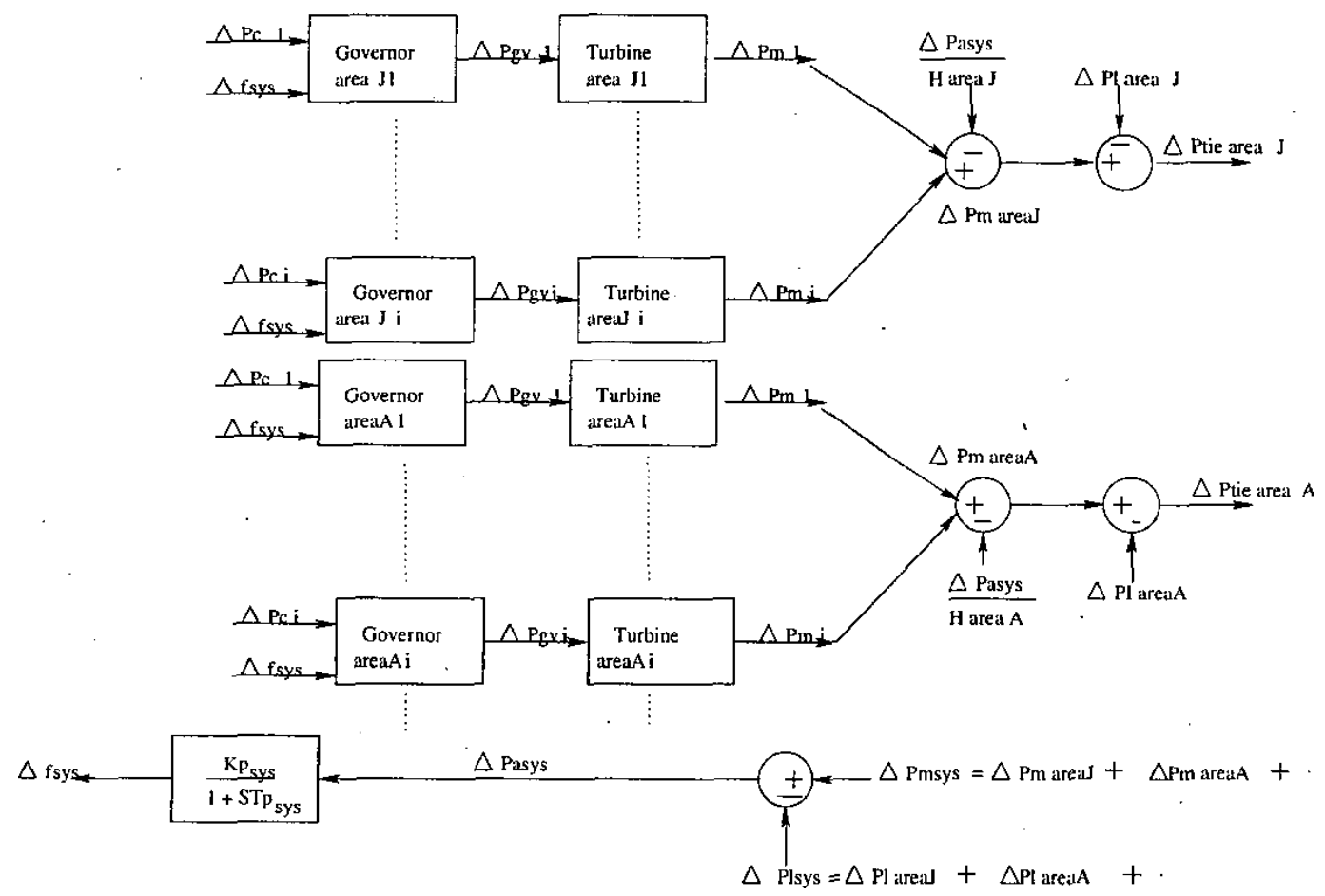

Figure 1. AGC simulation model : general structure

oscillations and electromechanical transients can he eliminated by the use of a single system frequency. A detailed investigation made in [3] also shows that such a simulation scheme indeed captures only the relevant slow dynamics filtering out the intermachine oscillations and electromechanical transients.

The main objective of any AGC simulation model is to compute the ACE defined in (1), which is a function of the tie line flow and system frequency deviations.

$$
A C E=B \text { Af sys }+\triangle \text { Ptiearea }_{A}
$$

In the present scheme the system frequency deviation is obtained by integrating the system accelerating power. The system accelerating power is computed from the system load and generation. The system generation is computed as the sum of individual unit generation, which is obtained by integrating each prime mover models (irrespective of the area in which they are present). The tie line flow deviation of each area is obtained by taking into account the area accelerating power, area load and area generation changes. Depending on the type of ACE signal (continuous or discrete) required, this can he computed either at every integration step or at the end of every AGC cycle. Based on the ACE, a suitable control action can he taken either continuously or discretely (at the end of every AGC cycle): The control action changes the set point ofthe governor.
A practical system consists of a number of prime mover units either hydro, non-reheat thermal or reheat thermal. From Fig 1 it is evident that for such a system, the computational complexity increases, since the model of each generator is to be integrated to get $\Delta P m$. Hence, an attempt is made here to reduce this effort by resorting to the use of the lower order models for these units [4].

\section{REDUCED ORDER PRIME MOVER MODELS}

A number of model order reduction techniques have been proposed in the control system literature. Some of these methods have been used earlier in power system stability studies. However, the use of model order reduction technique for AGC studies has not been investigsted earlier.

One of the earliest methods proposed for model order reduction (in the frequency domain) is the continuous fraction expansion (CFE) method [5]. This method is simple and it has been shown in [5] that it approximates the full order model very well in the low frequency region. In this method [6] the states are sorted in such a manner that the states having the least influence on the system response is at the end, while that dominant at the beginning of the state vector. Then the lower order model of a chosen order $(r)$ can be obtained by retaining the first $r$ state variables and discarding the others.

Recently the balanced realization method [7] has been widely used to obtain lower order models. This method takes a 


\section{Control Systems and Applications / 1101}

slightly different approach, because they are based on the input/output behavior of the system.--In this method, a realization is chosen for the system such that the input to state coupling and the state to output coupling are weighted equally so that state components which are weakly coupled to both the input and output are discarded. This method has the advantage of being computationally simple using standard matrix software, and it is possible to predict the error between the frequency response of the full and reduced order models. Another closely related approach is the Hankel norm model reduction [8] and [9], This is the only method where in the reduction technique is said to he optimal.

Table 1. Full and reduced order'prime mover models

\begin{tabular}{|l|l|}
\hline $\begin{array}{l}\text { Transfer } \\
\text { function }\end{array}$ & $\begin{array}{l}\text { Non-relieat } \\
\text { thermal }\end{array}$ \\
\hline Full order & $\frac{11.67}{(s+12.6000)(s+3.3339)}$ \\
CFE & $\frac{2.621}{s+2.632}$ \\
Balanced & $\frac{-0.1451 s+2.965}{s+2.965}$ \\
Hankel & $\frac{-0.07268+2.465}{s+2.298}$ \\
\hline
\end{tabular}

Table 2. Full and reduced order prime mover models

\begin{tabular}{|c|c|}
\hline $\begin{array}{l}\text { Transfer } \\
\text { function }\end{array}$ & $\begin{array}{l}\text { Reheat } \\
\text { thermal }\end{array}$ \\
\hline \multirow{6}{*}{$\begin{array}{l}\text { Full order } \\
\mathrm{CFE} \\
\text { Balanced } \\
\text { Hankel }\end{array}$} & $20.833(s+0.2)$ \\
\hline & $\begin{array}{c}(s+12.5000)(s+0.1000)(s+3.3333) \\
1.2864(s+0.200)\end{array}$ \\
\hline & $(s+2.5780)(s+0.1000)$ \\
\hline & $\frac{-0.0713(s+0.1990)(s-20.6509)}{(s+2.9348)(s+0.0999)}$ \\
\hline & $-0.0356(s+0.1341)(8-34.2966)$ \\
\hline & $(s+2.1919)(s+0.0721)$ \\
\hline
\end{tabular}

Transfer function representation of the Hydro unit model

$$
\frac{-0.4(s+0.2)(s-1)}{(s+0.0250)(s+2.0000)(s+1.9493)}
$$

The effectiveness of these model order reduction techniques in obtaining lower order prime mover models has been investigated here. The lower order models, for the above mentioned thermal prime mover models, have been obtained using the CFE, balanced and Hankel model order reduction techniques. The transfer function representation of the thermal prime mover models have been given in Table ?? and that of hydro has been given in (2). As the dynamics of the hydro unit could not be approximated satisfactorily by a lower model, the reduced order models for the thermal units alone have been obtained. The frequency responses of the reduced order models (obtained using the above 3 reduction techniques) match well with their full order models.

In order to provide a comparison of these approaches, the time response plots (for a step disturbance) of the reduced

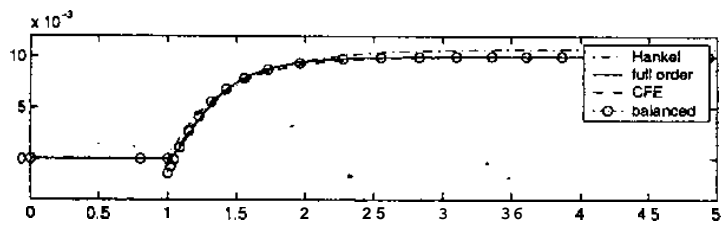

Figure 2. Time response plots : full and reduced order thermal nonreheat models

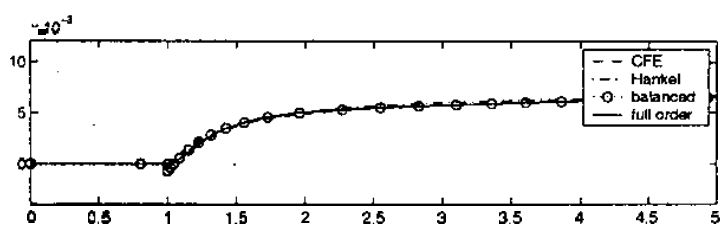

Flgure 3. Time response plots : full and reduced order thermal reheat models

as well as full order thermal non-reheat and reheat models are given in Fig 2 and 3. From these it can be seen that the response of the lower order models obtained using all the three methods (CFE balanced and Hankel) are comparable to that of the full order model. The time response plot of the lower order model (reheat as well as non-reheat) obtained using CFE technique seems to be identical to that of the full order model. As the CFE method is computationally simpler the use of this method to obtain lower order AGC models has been proposed here.

\section{REDUCED ORDER AGC MODELS}

The reduced order AGC models can be obtained by using the lower order prime mover models obtained by CFE method (for thermal reheat as well as non-reheat units) in the AGC model (Fig 1). In the systems where the hydro units are present they are represented by their full order models. The reliability of such models, for AGC studies, has been investigated here by considering a reasonably realistic test system (LEEE 30 bus test system). First, the primary response of the full and reduced order AGC models have been compared. Later, the use of these reduced order models for the AGC design has been investigated by designing an integral controller considering the reduced order model and studying its response with the full order model.

The IEEE 30 bus test system [10] has been divided in to three areas and the corresponding details are given in Table 3. From this it can be seen that some of the areas have different types of generating units.

\section{Response without the AGC}

A comparative study of the primary responses (without the AGC) of the full and reduced order AGC model for the above test system has been made. Fig $\mathbf{4}$ shows the variation in sys: tem frequency and tie line power flow of all the three areas, 
TENCON 2003/1102

Table 3. LEEE 30 bus test system

\begin{tabular}{|c|c|c|}
\hline Bus number & area number & $\begin{array}{l}\text { generator bus number } \\
\text { and type }\end{array}$ \\
\hline $\begin{array}{l}1-7,11-16 \\
17-20,24-30\end{array}$ & $\frac{I}{2}$ & 1,13-hydro; \\
\hline $87-20^{2} 24^{2}-30$ & 3 & 2. non-reheat \\
\hline $8-10,21-23$ & & $\begin{array}{l}27 \text {-reheat; } \\
22 \text { - non-reheat }\end{array}$ \\
\hline
\end{tabular}
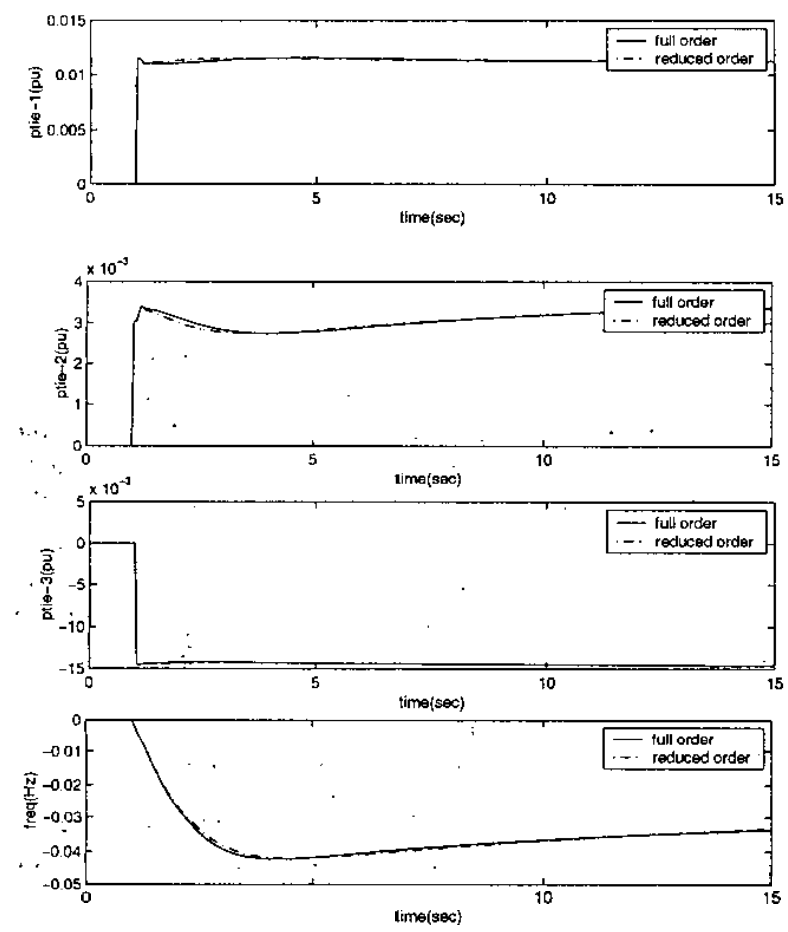

Figure.4. Variation in tie line flow and frequency : full and reduced order $\mathbf{A G C}$ models (IEEE 30 bus system)

for a $0.01 \mathrm{pu}$ step load disturbance in area-3, of the reduced and full order AGC model. From this it can be seen that the variation in-frequency and tie line power of all the three areas obtained using the full and reduced order models are identical. Thus it can be seen that the lower order models for thermal units can be used and full order of the hydro units can be used to obtain a satisfactory response, when various types of prime movers are present.

From this it can be concluded that the primary response of the reduced order AGC models is comparable with that of the full order model.

\section{Response of the integral controller}

The integral controller gain has been obtained by trial and error so as to reduce the peak overshoot and the response set-
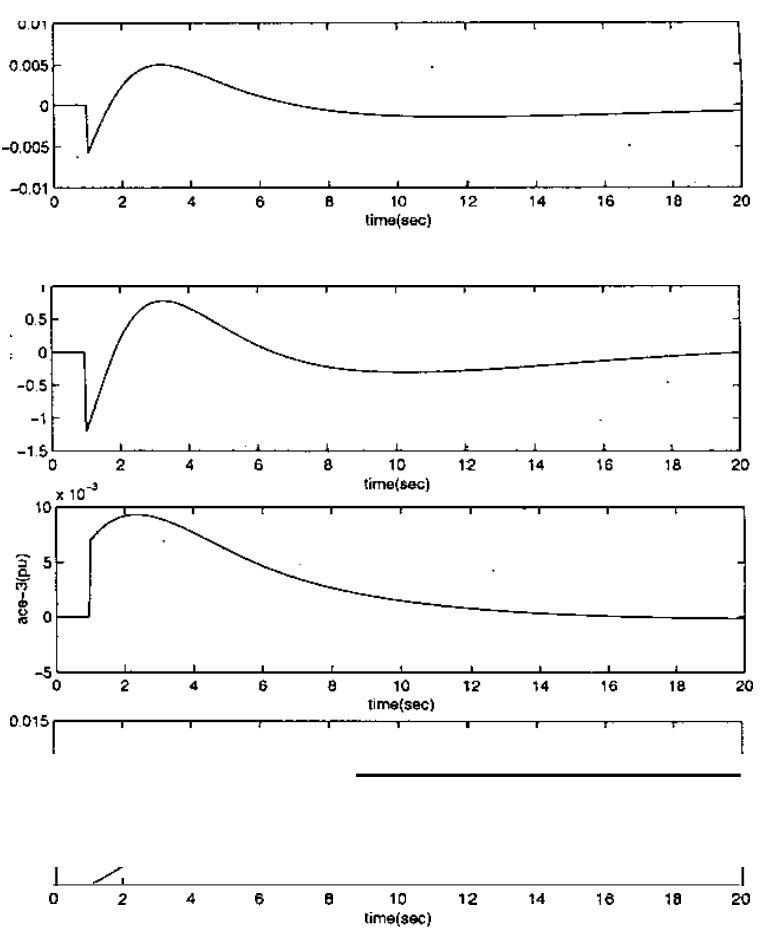

Figure 5. Integral controller response : full order AGC models (IEEE 30 bus system)

tling time. Even though various other design procedures are available to obtain optimal gain, they all require a quantitative specification of the control objective. But, there is no widely accepted performance specification for the AGC problem.

For this study, it is assumed that all the 6 generators of the above mentioned test system (Table $\mathbf{3}$ ) to be under AGC with an equal participation factor (i.e. 1/3, 1/2 and 1 for each generator of area-I, area-3 and area-2 respectively). A controller gain of 0.2 was found to be suitable, which is obtained by using the reduced order $\mathbf{A G C}$ model.

The integral controller response (with a gain of 0.2 ) of the full order AGC model for the above mentioned test system is shown in Fig 5. The response of the full order AGC model (with the integral controller) has been obtained for a $0.01 \mathrm{pu}$ step load disturbance (Fig 5). From this it can be seen that the $\mathbf{A G C}$ can be designed using the reduced order simulation model.

\section{Conclusion}

In this paper a novel AGC simulation model has been proposed. This model overcomes the major limitations of the existing model by assuming all the areas to be operating at the same frequency and by preserving the identity of each generating unit. Although this increases the computational effort, it can be reduced by using lower order models for the 
generating -units. The possibility of obtaining lower order models for the prime mover units has been shown by using CFE, balanced and Hankel model order reduction techniques - These reduced order prime mover models have been used to obtain the reduced order AGC model. The reduced order AGC models have been validated by comparing the primary response of these models with the full order models. Further, the amenability of these lower order models for AGC design has been shown by designing an integral controller.

\section{REFERENCES}

[1] T.M. Athay, "Generation scheduling and control," Proceedingsofthe IEEE, 75, 1592-1606, 1987.

[2] N. Jaleeli, L.S. Vanslyck, D.N. Ewart, L.H. Fink nnd A.G. Hoffmarn, "Understanding automatic generation control," IEEE Transactions on Power Systems, 7, , 106= 1121,1992 .

[3] Divya K.C.MSc. (Engg) thesis, Electrical Engg, Dept., I I Sc, Bangalore, India, 2002.

[4] IEEE. Committee, "Dynamic models for steam and hydro turbines in power systems studies," IEEE Transaclions on Power Apparatus and Systems, 92, 1904-1915, 1973.

[5] C.F. Chen and L.S. Shieh, "A novel approach to linear model simplification,"International Journal of Control, 8, 561-570, 1968.

[6] S.S. Lamba and S. Vittal Rao, "Aggregation matrix for the reduced order continued fraction expansion model of Chen and Shieh," IEEE Transactions on Automatic control, 23, 81-83, 1978.

[7] B.C. Moore, "Principal component analysis in linear systems: Controllability, Observability and model reduction," IEEE Transactions on Automatic control, 26, pp. 17-31,1981.

[8] L.M. Silverman and M. Bettayeb, "Optimal approximation of linear system," Proceedings JACC, San Francsico, CA, 1980.

[9] K. Glover K, "All optimal Hankel-norm approximation of linear multivariable systems and their $L^{\infty}$ error bounds," International Journal of Control, 39, 1115 $1193,1984$.

[10] Stagg and El-Abiad, Computer Methods in Power System Analysis, McGraw-Hill Book Company, 1968. 